transported to an area for a test or procedure, but once at their destination, patients are placed in an appropriately ventilated room if the organisms are airborne, such as the varicella zoster virus or M ycobacterium tuberculosis. Personnel then wear appropriate protective masks or respirators. Some may say that having the patient wear a mask is better than nothing, but I disagree. It simply provides a false sense of security that can be dangerous to others who are susceptible or at risk from the patient's infection. Based on this belief alone, the approach suggested by Haiduven et al is one we would not want to adopt.

Inge Gurevich, RN, MA Winthrop-University Hospital Mineola, New York

The authors reply.

Ms. Gurevich states, based on the belief that masks "provide a false sense of security," that she would not want to adopt the approach described in our article.

First of all, our article does not advocate the prolonged masking of patients. We do not mask patients for prolonged periods, but do mask them while they are transported to an area for a test or procedure or to an outside area for a family visit. However, we also would add that we believe that a mask is better than nothing. A barrier that can afford some degree of protection is certainly better than leaving it off to avoid a "false sense of security," thereby foregoing any protection. Even the article by Hoffsinger and Halpern, cited by Ms. Gurevich, illustrates in one of its tables that wearing no mask resulted in a considerably larger average colony count per plate than wearing any of the three masks studied. We therefore should agree to disagree on this point. Ms. Gurevich does not address the main emphasis of our approach, and we would like to briefly reiterate our policy:

- We do not allow employees with clinical varicella infection to work.

- In addition, exposed employees who may be incubating the disease are screened for symptoms and sent home should they feel ill for any reason.

- The employees are instructed to change their masks every hour and whenever moist, thus not allowing them to wear the same mask for long periods.

- The approach has been described as "an alternative one." Employees have the choice to stay home; they rarely do so. Ms. Gurevich does not state her alternative to our policy.

We hope that the readers who work in institutions with large numbers of varicella exposures or large numbers of nonimmune personnel or both will appreciate the approach we describe. It has been an effective alternative at our institution. The problem with current Centers for Disease Control recommendations is that many staff would be sent home for many days postexposure and yet not develop chickenpox, an approach that is neither practical nor cost-effective.

Donna Haiduven, BSN, MSN, CIC
David A. Stevens, MD, FACP
Carmen Hench, BSN, CIC
Santa Clara Valley Medical Center
San Jose, California

\title{
Risk of Ventilator-Associated Pneumonia May Be Reduced with Continuous Aspiration of Secretions
}

\section{by Gina Pugliese, RN, MS Medical News Editor}

Ventilator-associated pneumonia commonly occurs as a result of aspiration of colonized secretions, despite the presence of an endotracheal cuff. Dr. J. Valles and colleagues in Barcelona, Spain, evaluated the effect of continuous aspiration of subglottic secretions in patients in a medicalsurgical intensive care unit who required more than 3 days of intubation. In a randomized trial, study patients were intubated with an endotracheal tube that had a large opening on the dorsal side of the tube, just above the tracheal cuff, to allow for continuous aspiration. The cuff pressure was maintained at approximately $22 \mathrm{~mm}$ $\mathrm{Hg}$, and all patients received sucralfate. In addition to clinical and radio- graphic findings, a diagnosis of pneumonia was based on the results of quantitative cultures of bronchial brush catheters and bronchoalveolar lavage.

Fourteen $(18.4 \%)$ of 76 casepatients who received continuous aspiration developed pneumonia, compared to $25(32.5 \%)$ of 77 control patients. The incidence of ventilatorassociated pneumonia was 19.1 per 1,000 ventilator days in cases, compared to 39.6 per 1,000 days in the control group. Further, the onset of pneumonia was later in the continuously aspirated group compared to controls (12.0 days versus 5.9 days; $\mathrm{P}<.001)$. Only three episodes of pneumonia occurred during the first week in the cases, compared to 21 episodes of pneumonia in controls $(\mathrm{P}<.001)$. There also was a significant reduction in the incidence of pneumonia caused by gram-positive organisms in the continuously aspirated group, but no difference in pneumonia caused by gramnegative aerobic bacilli.

The author concluded that a simple modification in the endotracheal tube to allow continuous aspiration of secretions in mechanically ventilated patients may reduce the risk of pneumonia caused by gram-positive cocci and $\mathrm{H}$ aemophilus influenzae; the use of sucralfate also may play a role. It is unclear why there was not a significant difference in the risk of pneumonia due to gram-negative aerobic bacilli.

FROM: Valles J, et al. Continuous aspiration of subglottic secretions in preventing ventilator-associated pneumonia. A nn Intern M ed. 1995;122:179186. 